\title{
Didáctica invertida y las TIC: Un análisis de utilización en Educación Básica y Bachillerato
}

\section{Inverted Didactics and ICT: An analysis of utilization in Basic and Baccalaureate Education}

\author{
Fabiola Isabel Prieto-Urgilés \\ fabiola.prieto@psg.ucacue.edu.ec \\ Universidad Católica de Cuenca, Azogues \\ Ecuador \\ https://orcid.org/0000-0002-9632-5541 \\ Darwin Gabriel García-Herrera \\ dggarciah@ucacue.edu.ec \\ Universidad Católica de Cuenca, Azogues \\ Ecuador \\ https://orcid.org/0000-0001-6813-8100 \\ Sandra Elizabeth Mena-Clerque \\ sandramena@ucacue.edu.ec \\ Universidad Católica de Cuenca, Cuenca \\ Ecuador \\ https://orcid.org/0000-0002-9186-2161 \\ Juan Carlos Erazo-Álvarez \\ jcerazo@ucacue.edu.ec \\ Universidad Católica de Cuenca, Cuenca \\ Ecuador \\ https://orcid.org/0000-0001-6480-2270
}

Recibido: 20 de abril de 2020

Revisado: 05 de mayo de 2020

Aprobado: 26 de mayo de 2020

Publicado: 27 de junio de 2020 


\title{
RESUMEN
}

La investigación tuvo por objetivo determinar la efectividad de la didáctica basada en el aula invertida y TIC en educación básica y bachillerato del Ecuador, se procedió mediante un tipo descriptiva correlacional transversal con prueba de chi-cuadrado, lo cual permitió comprobar la asociación de las variables en estudio. Se determinó que el aula invertida o flip classroom no está siendo aplicada de una manera coherente con los referentes teóricos sobre el tema, lo cual trae consigo: El Aula Invertida no está cumpliendo con su propósito pues la mayoría de los docentes que usan esta metodología, asumen la praxis pedagógica del enfoque tradicional, lo cual refleja la no generación de un aprendizaje activo y reflexivo por parte del estudiante desde la investigación en apoyo de las TIC.

Descriptores: Aprendizaje activo; tecnología educacional;experimento educacional; educación alternativa. (Palabras tomadas del Tesauro UNESCO).

\begin{abstract}
The objective of the research was to determine the effectiveness of didactics based on the inverted classroom and ICT in basic education and high school in Ecuador. We proceeded by using a cross-sectional correlational descriptive type with a chi-square test, which allowed us to check the association of the variables in study. It was determined that the inverted classroom or flip classroom is not being applied in a manner consistent with the theoretical references on the subject, which brings with it: The Inverted Classroom is not fulfilling its purpose since most of the teachers who use this methodology, They assume the pedagogical praxis of the traditional approach, which reflects the non-generation of active and reflective learning by the student from research in support of ICT.
\end{abstract}

Descriptors: Activity learning; Educational technology; Educational experiments; Alternative education. (Words taken from the UNESCO Thesaurus).

\section{INTRODUCCIÓN}

El aula invertida en el contexto educativo ecuatoriano, es una tendencia nueva, pues el campo educacional se encuentra algo rezagado en el uso de recursos tecnológicos en educación básica, media, superior y bachillerato, el avance tecnológico está creciendo a pasos agigantados a nivel global, siendo oportuno actualizar a los docentes en este aspecto para innovar el proceso educativo, de este modo, incrementar las fortalezas y reducir los limitantes que implican el empleo de este enfoque. 
En Ecuador la Flipped Classroom, ha tomado relevancia en dos acontecimientos sociales que han repercutido en la educación. Estos hechos han obligado que los docentes, especialmente del nivel de educación general básica y bachillerato, adopten una metodología en la que el ciberespacio sea el escenario que favorezca la continuidad educativa. La problemática social surgida en octubre de 2019 en Ecuador por las diferentes protestas sociales, es el primer factor que influyó para que el (Ministerio de Educación MINEDUC, 2019), según su comunicado oficial de fecha siete de octubre de 2019, suspenda sus labores presenciales por ocho días en todos los establecimientos públicos, privados y fisco-misionales.

Por otra parte, mediante comunicado oficial de fecha catorce de marzo del dos mil veinte se da a conocer el Plan Educativo Covid-19 debido a la emergencia sanitaria mundial en la que las clases presenciales son suspendidas según comunicado oficial por parte del (MINEDUC, 2020). Este es el segundo acontecimiento que se ha generado un cambio en la forma en que los contenidos son impartidos por un lapso de tiempo que es aun indefinido.

Partiendo de lo planteado, (Wesley-Baker, 2016) señala que la finalidad del aula invertida es dotar al estudiante de material multimedia con contenidos relevantes para su aprendizaje con el fin que sea analizado y en caso de existir interrogantes, están sean aclaradas en clase, ya sea por los docentes que son mediadores o por sus pares mediante un aprendizaje colaborativo (Zaragoza-Martí, 2018); es así que se hace pertinente estudiar la operacionalización del Flipped Classroom como opción didáctica durante el tiempo de pandemia por COVID-19, de ese modo, la investigación tiene por objetivo determinar la efectividad de la didáctica basada en el aula invertida y TIC en educación básica y bachillerato del Ecuador.

\section{Referencial teórico}

A través de los años, grandes pedagogos como (Silva, 2018), nos han brindado un sin número de estrategias que favorecen a la dinamización del proceso de enseñanzaaprendizaje beneficiando al aprendizaje significativo y activo. Por esta razón, es menester dar una visión general de aquellos modelos educativos que renueven y refresquen aquellas metodologías que no hacen más que llevar a la frustración 
estudiantil pues priorizan la memorización y la pasividad de los estudiantes así como lo manifiesta (Muñoz-Morales, et al., 2019).

Con respecto a las metodologías que promueven el cambio pedagógico, (Luelmo-delCastillo, 2018) brinda una revisión de las Metodologías Activas, cuyo origen data con la aparición de las Escuelas Nuevas a finales del siglo XIX y su ideal fue transformar a la escuela tradicional que hacía que las personas sean seres pasivos y obedientes por una educación que forme a personas con espíritu crítico y solidario.

Así como lo manifiesta (Fernández-March, 2006) en su artículo metodologías activas para la formación de competencias, la lectura, escritura y solución de problemas son algunas de las actividades aplicadas en este método. Todo esto se logra a través de la interacción e intercambio de ideas y opiniones entre pares en el que el rol del docente es el de acompañar, apoyar y guiar al protagonista que es el estudiante que es quien resuelve problemas siendo reflexivo, crítico, colaborativo y respetuoso.

Una de las metodologías activas es el Aula Invertida que surge a partir de estos fundamentos al promover el aprendizaje activo y reflexivo. Los precursores de este enfoque son dos docentes estadounidenses (Bergmann \& Sams, 2014) que notaron que los estudiantes debido a la distancia del establecimiento y a las diversas actividades que ellos cumplían, no les era posible asistir de manera permanente a sus clases lo que causaba que se pase mayor tiempo de clases explicando nuevamente los contenidos dejando de lado el trabajo reflexivo de los estudiantes.

Esta situación permitió que se dé un giro, cuando el profesor Aarón Sams, leyó un artículo tecnológico sobre presentaciones de Power Point que podían ser modificadas de tal manera que tengan contenido visual y auditivo. De aquí surgió la idea de implementar esta estrategia y subirla al ciberespacio para ayudar a los estudiantes que no podían asistir a clases por algún factor, visualizar el material de clase desde cualquier lugar. La idea fue un éxito por cuanto poco a poco debido a que los tutoriales fueron puestos en línea tanto profesores como estudiantes de otros lugares los usaban para aclarar sus dudas sobre la materia (Bergmann y Sams, 2014)

Con respecto al punto antes mencionado, se debe considerar los pilares que (Rodríguez, 2017), considera como teoría para aplicar este método, mismos que toman su nombre de las iniciales de la palabra flip. Flexible environments, Learning 
culture, Intentional content and Professional educators. El primer punto demanda la flexibilidad y organización del tiempo y de un ambiente colaborativo por parte del docente para alcanzar un aprendizaje significativo. Por otra parte, es necesario que el estudiante tenga predisposición por aprender puesto que él es el protagonista de su formación y se requiere de una cultura de aprendizaje. Como tercera base está el contenido intencional, pues de los profesores depende clasificar conceptos y habilidades que lleven a la reflexión de los estudiantes. Finalmente, el rol del docente no puede desaparecer, pues es él quien guía y brinda de herramientas para el proceso educativo.

Llegados a este punto del análisis es necesario conocer los diversos puntos de vista que se han generado en torno al Aula al Revés. (Berenguer-Albaladejo, 2016) menciona que una de las ventajas que sugiere este método es la facultad con la que los estudiantes pueden generar un espíritu de compromiso y responsabilidad por aprender ya que pueden adaptar los conocimientos a su ritmo desarrollando su pensamiento analítico y crítico pues comparte su entendimiento con sus pares y en el caso de surgir alguna duda, esta es aclarada o guiada por su profesor quien posee más tiempo en el aula para aclarar dichas interrogantes

Por otro lado, entre las críticas que (Rodríguez, 2017) hace a este método es la conducta memorística y pasiva que los estudiantes deben mostrar hasta que sus incógnitas sean resueltas. Así mismo (Ramírez et al., 2014), sostienen que existen aún personas que no tienen acceso a recursos tecnológicos por tanto se genera una brecha de aprendizaje con aquellos que tienen la facilidad de obtener dichos aparatos. Por otro lado, (Plaza-de-la-Hoz, 2016) manifiesta que en algunos casos, el uso de dispositivos tecnológicos es un distractor por su mal direccionamiento y manejo. Como último punto en contra está el deseo de generar conocimientos reflexivos debido a que es una tarea fuera de clase y la decisión de hacerla o no significativa depende en gran parte del estudiante.

Con la idea anterior, es necesario realizar un análisis del ciberespacio como escenario en el Aula invertida es llevada a cabo. (Apolo et al., 2015), manifiestan que uno de los beneficios que ofrece la Web 2.0 se encuentra la infinidad de información y actividades a las que pueden acceder tanto los docentes como los dicentes. Con estos recursos 
se proveen actividades lúdicas, gráficas e innovadoras que favorecen el trabajo del aprendizaje moderno. De la misma manera, (Durán-Medina \& Vega-Baeza, 2013), mencionan la flexibilidad con la que se puede trabajar puesto que se puede establecer un aprendizaje asincrónico en donde la información puede ser visualizada y archivada con el fin de clarificar y ampliar el conocimiento en cualquier tiempo y lugar.

Otra de las ventajas que posee este escenario y como lo recomienda (HermannAcosta, 2011), es que la virtualidad permite la cooperación entre los actores educativos, puesto que se crea una interacción directa desarrollando una inteligencia colectiva. Por lo antes expuesto, el profesor pasa de ser expositor a ser mediador, mientras que el estudiante toma un rol reflexivo y hace suyo el conocimiento.

Por el contrario (Durán-Medina \& Vega-Baeza, 2013), exponen la ruptura del modelo tradicional con el que la mayoría de docentes trabaja en las aulas. A más de esto, la brecha digital que existe entre los dicentes y los docentes provocando que estos últimos eviten el uso de tecnologías con el fin de no sentirse opacados por los estudiantes quienes tienen un manejo natural de esta.

Asimismo, existen factores tanto externos como internos que influyen a que no sea posible el acceso y uso total de la tecnología. Dentro de los factores externos se encuentran la carencia de lugares o dispositivos tecnológicos a más del desconocimiento de las bondades que brindan los recursos virtuales. Los factores internos, por otro lado, tienen que ver con la pre-disposición por adquirir habilidades tecnológicas que favorezcan tanto la enseñanza como el aprendizaje (Martínez-Salas, 2019).

\section{MÉTODO}

La investigación al tener por objetivo determinar la efectividad de la didáctica basada en el aula invertida y TIC en educación básica y bachillerato del Ecuador, se procedió mediante un tipo descriptiva correlacional transversal con prueba de chi-cuadrado, lo cual permitió comprobar la asociación de las variables en estudio, así como la asociación lineal de los indicadores cualitativos y cuantitativos de la misma (SánchezUpegui, 2011). 
Se aplicó una encuesta de cohorte transversal mediante un cuestionario en escalamiento de Likert, de cinco alternativas de repuestas con resultado de coeficiente de Alfa de Cronbach de 0,94 a 60 docentes de la ciudad de Azogues-Ecuador, los cuales ejercen profesionalmente en diferentes subniveles y asignaturas del sistema educativo ecuatoriano (Hernández y Velasco-Mondragón, 2000).

Los datos recabados fueron analizados en su contexto natural y no se tuvo participación directa para modificar el concepto que los docentes tienen del Flipped Classroom, se desarrolló un diseño no experimental de investigación (MaldonadoGarcía, Erazo-Álvarez, Pozo-Cabrera, \& Narváez-Zurita, 2020).

\section{RESULTADOS}

Luego del análisis de los datos obtenidos en la presente encuesta, se han encontrado los siguientes resultados.

\section{Tabla 1}

Tabla de contingencia: Edad y empleo de dispositivos y programas multimedia por docentes

\begin{tabular}{|c|c|c|c|c|c|c|}
\hline & & & Emple & sitivos & & \\
\hline & & Bajo & Medio & Alto & Muy alto & \\
\hline & Recuento & 0 & 6 & 4 & 0 & 10 \\
\hline 安 & Frecuencia esperada & .7 & 3.8 & 4.8 & .7 & 10.0 \\
\hline స్ & $\%$ del total & $0.0 \%$ & $10.0 \%$ & $6.7 \%$ & $0.0 \%$ & $16.7 \%$ \\
\hline ח & Recuento & 1 & 7 & 11 & 3 & 22 \\
\hline 迆 & Frecuencia esperada & 1.5 & 8.4 & 10.6 & 1.5 & 22.0 \\
\hline 이 & $\%$ del total & $1.7 \%$ & $11.7 \%$ & $18.3 \%$ & $5.0 \%$ & $36.7 \%$ \\
\hline "s & Recuento & 3 & 8 & 9 & 1 & 21 \\
\hline 迩 & Frecuencia esperada & 1.4 & 8.1 & 10.2 & 1.4 & 21.0 \\
\hline F & $\%$ del total & $5.0 \%$ & $13.3 \%$ & $15.0 \%$ & $1.7 \%$ & $35.0 \%$ \\
\hline $\mathscr{n}$ & Recuento & 0 & 2 & 5 & 0 & 7 \\
\hline 定 & Frecuencia esperada & .5 & 2.7 & 3.4 & .5 & 7.0 \\
\hline 品 & $\%$ del total & $0.0 \%$ & $3.3 \%$ & $8.3 \%$ & $0.0 \%$ & $11.7 \%$ \\
\hline
\end{tabular}


Fabiola Isabel Prieto-Urgilés; Darwin Gabriel García-Herrera; Sandra Elizabeth Mena-Clerque; Juan Carlos Erazo-Álvarez

\begin{tabular}{|c|c|c|c|c|c|}
\hline Recuento & 4 & 23 & 29 & 4 & 60 \\
\hline Frecuencia esperada & 4.0 & 23.0 & 29.0 & 4.0 & 60.0 \\
\hline$\%$ del total & $6.7 \%$ & $38.3 \%$ & $48.3 \%$ & $6.7 \%$ & $100.0 \%$ \\
\hline
\end{tabular}

\begin{tabular}{|c|c|c|c|}
\hline & Valor & gl & Sig. asintótica (bilateral) \\
\hline Chi-cuadrado de Pearson & $8.663^{a}$ & 9 & 0.469 \\
\hline Razón de verosimilitudes & 9.894 & 9 & 0.359 \\
\hline Asociación lineal por lineal & .002 & 1 & 0.960 \\
\hline N de casos válidos & 60 & & \\
\hline
\end{tabular}

Fuente: Elaboración propia

En la primera tabla, el nivel de significancia del $0.05 \%$ y con 9 grados de libertad según la tabla de Chi-cuadrado de Pearson, obteniendo un valor límite de 8,663 es decir una significancia asintótica (bilateral) de 0.469 misma que es corroborada con las medidas simétricas. Los resultados que se encuentran en este rango, muestran que debido a que el resultado es mayor al nivel de significancia la hipótesis es nula; es decir, la edad de los docentes no repercute en la habilidad para emplear la tecnología.

\section{Prueba de hipótesis 1}

\section{Hipótesis}

H0: La edad de los docentes no repercute en la habilidad para emplear la tecnología. H1: La edad de los docentes repercute en la habilidad para emplear la tecnología. Se acepta $\mathrm{H} 0$ y se desecha $\mathrm{H} 1$.

\section{Tabla 2}

Tabla de Contingencia: Empleo de dispositivos y programas multimedia y Conocimiento previo a una clase.

\begin{tabular}{|c|c|c|c|c|c|c|c|c|}
\hline & & & Conc & cimien & to previc & a una & clase & \\
\hline & & & $\begin{array}{l}\text { Muy } \\
\text { bajo }\end{array}$ & Bajo & Medio & Alto & $\begin{array}{l}\text { Muy } \\
\text { alto }\end{array}$ & Total \\
\hline Empleo de & & Recuento & 1 & 0 & 2 & 0 & 1 & 4 \\
\hline dispositivos y & Bajo & Frecuencia esperada & 0.3 & 0.7 & 1.7 & 1.1 & .2 & 4.0 \\
\hline programas & & $\%$ del total & $1.7 \%$ & $0.0 \%$ & $3.3 \%$ & $0.0 \%$ & $1.7 \%$ & $6.7 \%$ \\
\hline
\end{tabular}


Fabiola Isabel Prieto-Urgilés; Darwin Gabriel García-Herrera; Sandra Elizabeth Mena-Clerque; Juan Carlos Erazo-Álvarez

multimedia

$\begin{array}{ll}\text { Medio } & \text { Recuento } \\ & \text { Frecuencia esperada } \\ & \text { Recuento } \\ \text { Alto } \quad & \text { Frecuencia esperada } \\ & \% \text { del total } \\ & \text { Recuento } \\ \text { Muy alto } & \text { Frecuencia esperada } \\ & \% \text { del total } \\ & \text { Recuento } \\ \text { Total } \quad \text { Frecuencia esperada } & \text { \% del total }\end{array}$

$\begin{array}{cccccc}1 & 6 & 12 & 3 & 1 & 23 \\ 1.9 & 3.8 & 9.6 & 6.5 & 1.2 & 23.0 \\ 1.7 \% & 10.0 \% & 20.0 \% & 5.0 \% & 1.7 \% & 38.3 \% \\ 3 & 4 & 8 & 13 & 1 & 29 \\ 2.4 & 4.8 & 12.1 & 8.2 & 1.5 & 29.0 \\ 5.0 \% & 6.7 \% & 13.3 \% & 21.7 \% & 1.7 \% & 48.3 \% \\ 0 & 0 & 3 & 1 & 0 & 4 \\ 0.3 & 0.7 & 1.7 & 1.1 & 0.2 & 4.0 \\ 0.0 \% & 0.0 \% & 5.0 \% & 1.7 \% & 0.0 \% & 6.7 \% \\ 5 & 10 & 25 & 17 & 3 & 60 \\ 5.0 & 10.0 & 25.0 & 17.0 & 3.0 & 60.0 \\ 8.3 \% & 16.7 \% & 41.7 \% & 28.3 \% & 5.0 \% & 100.0 \%\end{array}$

\begin{tabular}{|c|c|c|c|}
\hline & Valor & Gl & $\begin{array}{c}\text { Sig. asintótica } \\
\text { (bilateral) }\end{array}$ \\
\hline Chi-cuadrado de Pearson & $17.461^{a}$ & 12 & 0.133 \\
\hline Razón de verosimilitudes & 18.331 & 12 & 0.106 \\
\hline Asociación lineal por lineal & 0.930 & 1 & 0.335 \\
\hline $\mathrm{N}$ de casos válidos & 60 & & \\
\hline
\end{tabular}

Fuente: Elaboración propia

Estos resultados fueron obtenidos mediante el análisis Chi-cuadrado de Pearson, se obtiene una significancia asintótica de 0.133 misma que se encuentra en un valor mayor al nivel de significancia de $0,05 \%$ dando como resultado una hipótesis nula en el que se manifiesta la falta de conocimientos previa a una clase.

\section{Prueba de hipótesis 2}

\section{Hipótesis}

HO: Los docentes consideran que los estudiantes no poseen conocimientos previos a una clase

H1: Los docentes consideran que los estudiantes sí poseen conocimientos previos a una clase

Se acepta $\mathrm{H} 0$ y se rechaza $\mathrm{H} 1$ 


\section{Tabla 3}

Tabla de contingencia: Percepción docente sobre aula invertida y la frecuencia de investigación estudiantil previa sobre un tema nuevo de clase.

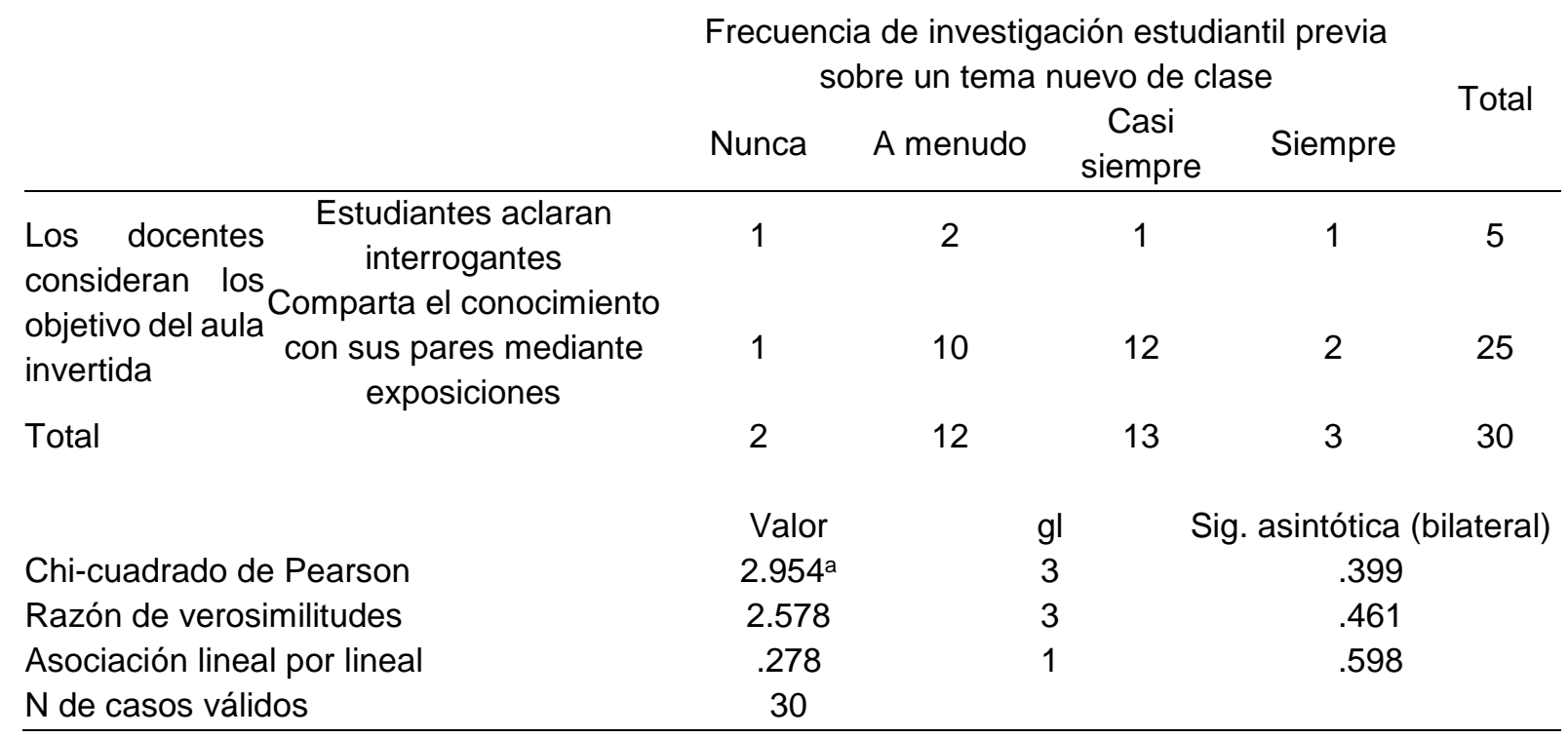

Fuente: Elaboración propia

No sólo los datos mostrados anteriormente brindan información relevante para la investigación sino también la siguiente tabla nos indica nuevamente una hipótesis nula al mostrar una significancia asintótica de 0.399 cuya hipótesis muestra que no existe reflexión cuando se emplea el Aula Invertida a pesar que existe investigación previa.

\section{Prueba de hipótesis 3}

\section{Hipótesis}

H0: No se genera reflexión cuando se usa el aula invertida a pesar de existir investigación previa por parte de los estudiantes.

H1: Se genera reflexión cuando se usa el aula invertida a pesar de existir investigación previa por parte de los estudiantes.

Se acepta $\mathrm{HO}$ y se rechaza $\mathrm{H} 1$. 


\section{DISCUSIÓN}

Prueba de hipótesis 1, contradice lo afirmado por Durán-Medina y Vega-Baeza (2013) quienes exponen que los profesores evitan el uso de tecnología para evitar quedarse rezagados de la habilidad del manejo de las mismas por parte de los estudiantes. No obstante, con estos resultados la afirmación de Martínez-Salas (2019) tiene validez ya que los factores internos o la predisposición de los maestros por emplear tecnología favorecen al proceso de aprendizaje pues no depende de la brecha tecnológica el uso adecuado de estos recursos.

La prueba de hipótesis 2, demuestra que el Aula invertida no está siendo empleada de manera adecuada pues autores como Bergmann \& Sams (2014), Wesley-Baker (2016), Berenguer-Albaladejo (2016), entre otros, coinciden en que la finalidad del Aula Invertida es proveer a los estudiantes de material previo a recibir una clase pues en el aula las dudas o inquietudes que surgen del análisis de dicho material es aclarado mediante la participación y reflexión de los contenidos previamente analizados.

La prueba de hipótesis 3, contradice lo planteado por (Peche-Cruz \& Giraldo-Supo, 2019), quienes indican que el aula invertida permite generar reflexión en los estudiantes, por cuanto se requiere el procesamiento de búsqueda de información, interpretación, análisis, comprensión, lo cual permite configurar una postura reflexiva desde la educación frente a los diversos hechos sociales a los cuales se enfrenta el discente.

Por otro lado, (Hernández-Silva \& Tecpan-Flores, 2017), concuerdan que el aula invertida está organizada de tal manera en la que se da un giro al proceso de clase. Es decir, primeramente, se envía las tareas a los estudiantes para que sean analizadas y reflexionadas en casa y luego construir el conocimiento cuyo medio es la cooperación entre pares siendo el docente un mediador (Quevedo-Álava, et al., 2020).

En un análisis general, se deduce que para los educadores el factor tecnológico no es un impedimento para crear material multimedia que favorece la socialización de contenidos valiosos para los estudiantes. Sin embargo, el rol de los docentes se debe 
enfocar en motivar de cierto modo a los estudiantes como lo sugiere (HermannAcosta, 2011) para que los datos que los estudiantes reciben no sean acumulados sin sentido, más bien la información sea analizada y reflexionada.

Todos estos datos señalan que por el momento el aula invertida no cumple su propósito por percepciones que los docentes tienen acerca de esta metodología. Por un lado, se encuentran aquellos docentes que suponen que el aula invertida es implementar recursos tecnológicos a sus clases que siguen teniendo un enfoque pasivo pues los estudiantes son simplemente oyentes y de esta forma el tiempo promedio de clase que es entre 40 o 60 minutos es empleada en dar una clase nueva con la participación mínima de los estudiantes (Arroyo-Carrera, et al., 2020).

Por otro lado, cuando existe investigación previa, esta es realizada de manera autónoma por parte de los estudiantes por cuanto el profesor simplemente da un tema general a ser indagado mismo que debe ser expuesto posteriormente en clase y a sus compañeros. En este apartado, la concepción que los docentes tienen del aula invertida es la de cambiar roles con los estudiantes (Argandoña-Mendoza, et al., 2020).

Todos estos resultados se alejan de la aplicación correcta del Aula invertida debido a que los educadores se están limitando a deducir o a interpretar su nombre mas no se analiza la esencia de esta metodología que dicho de varios autores favorece al aprendizaje significativo y colaborativo (Chirino-García \& Hernández-Corona, 2020).

\section{PROPUESTA}

La actualización docente es una necesidad que debe ser priorizada con el fin de innovar el proceso pedagógico, adaptándose a una nueva realidad con la finalidad de contribuir a la formación de los educandos ya que la educación y virtualidad forman parte de la naturaleza estudiantil hoy en día. Por lo antes mencionado, el Aula Invertida permite que el ciberespacio sea empleado en la educación, permitiendo que este proceso sea dinámico y se adapte a la realidad actual.

Con el fin de que esta metodología sea aplicada adecuadamente se plantean algunos lineamientos que optimicen la aplicación de la misma (Figura 1). 


\section{Concientizar}

1.1 ¿Cuál es es objetivo detrás del Aula Invertida?

\section{Guiar}

2.1 Dar a conocer las herramientas tecnológicas usadas par la creación de material virtual.

\section{Implementar}

3.1 Aplicación del Aula Invertida en el proceso educativo.

\begin{tabular}{l} 
4. Evaluar \\
$\begin{array}{l}4.1 \text { Examinar los cambios actitudinales generados en los } \\
\text { educandos. }\end{array}$ \\
\hline
\end{tabular}

Figura 1. Propuesta utilización aula invertida. Fuente: Elaboración propia

1. Concientizar: La creación de espacios de discusión en la que se compartan experiencias sobre el uso del Aula Invertida es esencial para aclarar dudas o malinterpretaciones sobre este enfoque ya que como se observó en los resultados el desconocimiento de su finalidad es común entre los docentes. Otra opción que se puede aplicar en este paso es la creación de podcasts en los que se incluya la información relevante sobre esta metodología para que de esta manera el mensaje sea difundido en diferentes medios sociales. 
2. Socializar: Generar tutoriales en los que se dé a conocer las diferentes utilidades que se puede dar a los recursos empleados para la creación de material visual ya sean presentaciones, aulas virtuales, generador de textos, redes sociales, entre otros. Todo con la finalidad de facilitar la divulgación de contenidos previo a una clase cumpliendo de esta manera el objetivo del Flipped Classroom. En este punto, la ventaja que se presenta es la predisposición que los docentes muestran por aprender algo nuevo y no quedarse rezagados en el uso de la tecnología.

3. Implementar: Una vez que el docente tiene el conocimiento teórico-práctico del aula invertida, ya puede ser aplicada. En el modelo en mención, si los estudiantes tienen acceso previo al conocimiento de temas que serán analizados en clase, el proceso se dinamiza puesto que en clase quienes construyen los saberes son ellos mediante el intercambio de ideas, resolución de conflictos, entre otros. En este punto, el rol del docente cambia ya que pasa de ser la única persona que dominaba los temas tratados, a ser un guía que aclara interrogantes e impulsa el autoconocimiento favoreciendo de esta manera el aprendizaje.

4. Evaluar: Para finalizar, es necesario conocer si se está dando un cambio sustancial luego de la aplicación de esta metodología activa. Es decir, mediante conversatorios o la aplicación de encuestas descriptivas a los estudiantes, se pueden obtener datos que muestren el efecto que produce este enfoque.

\section{CONCLUSIONES}

Se determinó que el aula invertida o flip classroom no está siendo aplicada de una manera coherente con los referentes teóricos sobre el tema, lo cual trae consigo: El Aula Invertida no está cumpliendo con su propósito pues la mayoría de los docentes que usan esta metodología, asumen la praxis pedagógica del enfoque tradicional, lo cual refleja la no generación de un aprendizaje activo y reflexivo por parte del estudiante desde la investigación en apoyo de las TIC. 


\section{FINANCIAMIENTO}

No monetario.

\section{AGRADECIMIENTO}

A la Universidad Católica de Cuenca por el apoyo en la realización de la investigación.

\section{REFERENCIAS CONSULTADAS}

Argandoña-Mendoza, M., García-Mejía, R., Ayón-Parrales, E., \& ZambranoZambrano, Y. (2020). Investigación e innovación educativa: Reto escolar por COVID-19 en el Ecuador. [Educational research and innovation: School challenge for COVID-19 in Ecuador]. EPISTEME KOINONIA, 3(5), 162-182. http://dx.doi.org/10.35381/e.k.v3i5.726

Arroyo-Carrera, E., Loor-Santos, M., Mendoza-Mera, J., \& Solorzano-Zambrano, M. (2020). Gestión de aprendizaje creativo mediante la Herramienta Powtoon en estudiantes de lengua y literatura. [Creative learning management through the Powtoon Tool in language and literature students]. EPISTEME KOINONIA, 3(5), 251-267. http://dx.doi.org/10.35381/e.k.v3i5.775

Apolo, D., Bayés, M., \& Hermann, A. (2016). Cambios educativos en los procesos de lectura digital: la pedagogía del ciberespacio como estrategia de procesamiento de contenidos en la era de internet. [Educational changes in the processes of digital reading: the pedagogy of cyberspace as a content processing strategy in the internet age]. Revista De Estudios Para El Desarrollo Social De La Comunicación, 0(12), 222. doi:10.15213/redes.n12.p222

Berenguer-Albaladejo, C. (2016). Acerca de la utilidad del aula invertida o flipped classroom. [About the usefulness of the flipped classroom]. Recuperado de https://n9.cl/ylvh9

Bergmann, J., \& Sams, A. (2014). Dale la vuelta a tu clase. [Turn your class]. Santa María-Ediciones. 13-23. Recuperado de https://n9.cl/egn8

Chirino-García, R., \& Hernández-Corona, J. (2020). M-learning: Estrategia para la promoción del aprendizaje electrónico móvil en instituciones de educación superior. [M-learning: Strategy for the promotion of mobile e-learning in higher education institutions]. EPISTEME KOINONIA, 3(5), 102-121. http://dx.doi.org/10.35381/e.k.v3i5.684

Durán-Medina, F., \& Vega-Baeza, R. (2013). El ciberespacio y la educación: ¿una pedagogía de la rentabilidad? Debilidades y fortalezas. Estudios sobre el Mensaje Periodístico. [Cyberspace and education: a pedagogy of profitability? Weaknesses and strengths]. Recuperado de https://n9.cl/vwymv 
Fernández-March, A. (2006). Metodologías activas para la formación de competencias. [Active methodologies for skills training]. Educatio Siglo XXI, 24, 35-56. Recuperado de https://n9.cl/ogxo

Hermann-Acosta, A. (2011). Pedagogía del ciberespacio: hacia la construcción de un conocimiento colectivo en la sociedad roja. [Cyberspace pedagogy: towards the construction of collective knowledge in the red society]. Sophia, Colección de Filosofía de la Educación, (11), 83-103. Recuperado de https://n9.cl/5l6p

Hernández-Silva, C, \& Tecpan-Flores, S. (2017). Aula invertida mediada por el uso de plataformas virtuales: un estudio de caso en la formación de profesores de física. [Inverted classroom mediated by the use of virtual platforms: a case study in the training of physics teachers]. Estudios pedagógicos (Valdivia), 43(3), 193-204. https://dx.doi.org/10.4067/S0718-07052017000300011

Hernández, B., \& Velasco-Mondragón, H. (2000). Encuestas transversales. [Crosssectional surveys]. Salud pública de México, 447-455. Recuperado de https://n9.cl/8fm68

Luelmo-del-Castillo, M. (2018). Origen y desarrollo de las metodologías activas dentro del sistema educativo español. [Origin and development of active methodologies within the Spanish educational system]. Encuentro: Revista de investigación e innovación en la clase de idiomas, 4-21. Recuperado de https://n9.cl/xbww

Maldonado-García, V., Erazo-Álvarez, J., Pozo-Cabrera, E., \& Narváez-Zurita, C. (2020). Violencia económica y patrimonial. Acceso a una vida libre de violencia a las mujeres. [Economic and patrimonial violence. Access to a life free of violence against women]. IUSTITIA SOCIALIS, 5(8), 511-526. http://dx.doi.org/10.35381/racji.v5i8.588

Martínez-Salas, M. (2019). El modelo pedagógico de clase invertida para mejorar el aprendizaje del idioma inglés. [The inverted class pedagogical model to improve English language learning]. Investigación Valdizana, 204-213. Recuperado de https://n9.cl/zo8gh

MINEDUC (2020). El Ministerio de Educación informa ante las nuevas decisiones tomadas por el Comité de Operaciones de Emergencia Nacional COE. [The Ministry of Education reports on new decisions made by the National Emergency Operations Committee COE]. Recuperado de https://n9.cl/taop2

Ministerio de Educación (2019). Suspensión de las actividades académicas para los estudiantes para precautelar la salud de la comunidad educativa. [Suspension of academic activities for students to protect the health of the educational community]. Recuperado de https://n9.cl/w1bp 
Muñoz-Morales, N., Barrientos-Oradini, N., Araya-Castillo, L., \& Reyes-Saavedra, J. (2019). Capacidades metacognitivas en el sistema educativo en instituciones educativas de educación media. [Metacognitive capacities in the educational system in educational institutions of secondary education]. Revista Arbitrada Interdisciplinaria Koinonía, 4(7), 103-127. http://dx.doi.org/10.35381/r.k.v4i7.196

Peche-Cruz, H., \& Giraldo-Supo, V. (2019). El Aprendizaje Flip Learning centrado en el estudiante como generador de calidad educativa. [Student-centered Flip Learning as a generator of educational quality]. Revista Arbitrada Interdisciplinaria Koinonía, 4(8), 427-450. http://dx.doi.org/10.35381/r.k.v4i8.293

Plaza-de-la-Hoz, J. (2016). Ventajas y desventajas del uso adolescente de las TIC: visión de los estudiantes. [Advantages and disadvantages of adolescent use of ICT: students' vision]. Complutense de Educación, 491-508. Recuperado de https://n9.cl/166w

Quevedo-Álava, R., Corrales-Moreno, L., Palma-Delgado, G., \& Mendoza-Suárez, G. (2020). Psicopedagogía y TIC en período de COVID-19. Una reflexión para el aprendizaje significativo. [Psychopedagogy and ICT in the COVID-19 period. A Reflection for Meaningful Learning]. EPISTEME KOINONIA, 3(5), 202-220. http://dx.doi.org/10.35381/e.k.v3i5.769

Ramírez, D., Hinojosa, C., \& Rodríguez, F. (2014). Advantages and disadvatages of flipped classroom: stem students' perceptions. [ventajas y desventajas del aula flipped: Percepciones de estudiantes stem]. Recuperado de https://cutt.ly/MiWCsL7

Rodríguez, W. (2017). Ideas y reflexiones para comprender la metodología Flipped Classroom. [Ideas and reflections to understand the Flipped Classroom methodology]. Revista Virtual Universidad Católica del Norte, 143-161. Recuperado de https://cutt.ly/wiWCGpM

Sánchez-Upegui, A. (2011). Manual de redacción académica e investigativa: cómo escribir, evaluar y publicar artículos. [Academic and research writing manual: how to write, evaluate and publish articles]. Católica del norte Fundación Universitaria, 165-172. Recuperado de https://cutt.ly/piWVxNU

Silva, C. (2018). Formación integral para facilitadores. [Comprehensive training for facilitators]. EPISTEME KOINONIA, $1(1), \quad 35-50$. http://dx.doi.org/10.35381/e.k.v1i1.489

Wesley-Baker, J. (2016). The origins of The classroom flip. [Los orígenes del aula invertida]. 15-23. Recuperado de https://cutt.ly/siWVOAP 
Zaragoza-Martí, M. F. (2018). El Flip Teaching como herramienta TIC en el proceso de enseñanza-aprendizaje jurídico-criminológico mediante el uso de la plataforma Moodle. [Flip Teaching as an ICT tool in the legal-criminological teaching-learning process using the Moodle platform]. Recuperado de https://cutt.ly/OiWBiny 